\title{
The current state and prospects for meteors observations in RI NAO
}

\author{
A. Shulga, N. Kulichenko, V. Vovk, Y. Kozyryev and Y. Sybiryakova \\ RI "Nikolaev Astronomical Observatory" Mykolaiv, 54030, Ukraine \\ email: avshulga@mail.ru
}

The observations of meteors using optical telescopes equipped with TV-cameras and the observations in radio signals using FM radio and TV translators are started in RI NAO since 2010.

The main purpose of observations is

- in optics - statistics of meteors at night time, the calculation of flux maximum, the calculation of the coordinates of the radiant flux,

- in the radio range - statistics of meteors within 24 hours, the calculation of flux maximum, and its width, the definition of the velocity of meteor.

Optical observations of meteors at the RI NAO are conducted using Meteor patrol, which includes two optical telescopes $(\mathrm{D}=47 \mathrm{~mm}, \mathrm{~F}=85 \mathrm{~mm})$ equipped with a TV CCD cameras WAT-902H2 $(768 \times 576,8.6 \times 8.3 \mu)$. The field of view of each telescope is $4.2^{\circ} \times 3.2^{\circ}$.

The process of observation and coordinate-photometric processing is automated using original software developed by the RI NAO. Software implements the method for automatical detecting of meteors using the search of cell of its image according signal/noise ratio which exceeds the liminal value on previous frames. In addition to the signal/noise ratio, the velocity of objects that allows not to register objects, such as satellites, airplanes, birds, and noises of CCD camera is also analyzed.

In 2011-2012, regular observations were conducted at the meteor patrol in RI NAO, more than 1200 meteors with the longitude of $(0.5-4.5)^{\circ}$ and brightness $(1-5) \mathrm{m}$ were recorded. The error of reference system is (1-6)". Error in determining of meteor trajectories arc in right ascension and declination was (10-12)". Error estimate of the pole of a large circle of the meteor trajectory was (3-13)'.

In the radio band the observations of meteors are carried out by detecting the horizon FM radio signal, which is reflected from the meteor trail. FM station in Kielce (Poland) is used as signal source.

Hardware and software package is designed. It includes a highly directional antenna, PC with TV/FM-tuner and software for automatic registration of meteors. Automatic extraction of fragments of audio signal of FM radio stations in the time-frequency space using the FFT algorithm is used. The original software is developed by the NAO.

The data processing of observations all of the data is carried out and more than 100,000 meteoric phenomena are given. The characteristic bursts of meteors for all major meteor showers are detected from the time series of observations.

A comparison of data of automatic processing of radio observations in the NAO with observations of meteors by video observations of meteors IMO network were conducted, the correlation coefficient was $>0.5$. 